\title{
The CDF dijet excess from intrinsic quarks
}

\author{
Xiao-Gang $\mathrm{He}^{1,2,3, \mathrm{a}}$ and Bo-Qiang $\mathrm{Ma}^{1, \mathrm{~b}}$ \\ 1 School of Physics and State Key Laboratory of Nuclear Physics and Technology, Peking University, Beijing 100871 \\ 2 Department of Physics and Center for Theoretical Sciences, National Taiwan University, Taipei 10617 \\ 3 Institute of Particle Physics and Cosmology, Department of Physics, Shanghai JiaoTong University, Shanghai 200240
}

Received: 5 November 2011 / Revised: 26 November 2011

Published online: 14 December 2011

(C) The Author(s) 2011. This article is published with open access at Springerlink.com

Communicated by B. Ananthanarayan

\begin{abstract}
The CDF Collaboration reported an excess in the production of two jets in association with a $W$. We discuss constraints on possible new particle state interpretations of this excess. The fact of no statistically significant deviation from the SM expectation for $Z+$ dijet events in CDF data disfavors the new particle explanation. We show that the nucleon intrinsic strange quarks provide an important contribution to the $W$ boson production in association with a single top quark production. Such $W+\mathrm{t}$ single top quark production can contribute to the CDF $W+$ dijet excess, thus the nucleon intrinsic quarks can provide a possible explanation to the CDF excess in $W+$ dijet but not in $Z+$ dijet events.
\end{abstract}

Recently, the CDF Collaboration reported an excess in the production of two jets in association with a $W$ boson production [1], in the Fermilab Tevatron collider of proton and antiproton collision at a center-of-mass energy of $1.96 \mathrm{TeV} / c^{2}$ with an integrated luminosity of $4.3 \mathrm{fb}^{-1}$ The $W$ boson is identified through a charged lepton (electron or muon) with large transverse momentum, and the invariant mass of the two-jet system $\left(M_{j j}\right)$ is found to be in the range of $120-160 \mathrm{GeV} / c^{2}$. The two-jet system appears to be an unidentified resonance with mass around $150 \mathrm{GeV} / c^{2}$, and this triggers the speculations to understand the dijet as a non-standard new particle resonance with the associated $W$ production cross-section to be about $4 \mathrm{pb}$. It is also reported [1] that such an excess of dijet events cannot be described within the statistical and systematic uncertainties of current theoretical predictions. Therefore the CDF excess has the potential of being an indication for new physics beyond the standard model (SM).

The purpose of this letter is to make an analysis of some possible explanations for the CDF dijet excess within and beyond the SM. We analyse constraints on the properties of the speculated Higgs or $Z^{\prime}$ resonance, if one tries to explain the CDF dijet excess as a signal from a new kind of particles beyond SM. The fact that no statistically significant deviation from the SM expectation for $Z$ plus dijets events disfavors new particle resonance explanation [2]. This leads us to go back to examine uncertainties con-

a e-mail: hexg@phys.ntu.edu.tw

b e-mail: mabq@pku.edu.cn cerning strange and heavy quark content of the nucleon. We show that some intriguing features of the nucleon sea, which are relevant to understand several anomalies previously found in experiments, can also provide some understanding for the newly reported CDF dijet excess.

There are many possible ways a new particle can manifest itself in theoretical models. Here we briefly discuss some properties of a beyond SM Higgs boson and a new vector gauge boson with a mass of $150 \mathrm{GeV} / c^{2}$ for the explanation of the CDF $W+$ dijet excess.

The CDF $W+$ dijet excess has been shown to be not compatible with SM $W H$ production, i.e., interpreting the dijet being from the decay of a SM Higgs with a mass of $150 \mathrm{GeV} / c^{2}$. The main reason is that the production crosssection $\sigma$ multiplied with the branching ratio $\mathrm{BR}(H \rightarrow b \bar{b})$ of a Higgs decays into a pair of $b \bar{b}$ is only about $12 \mathrm{fb}$ which would not give excess for $W H \rightarrow l \nu b \bar{b}$. This has been confirmed by the dedicated CDF search [2].

When going beyond the SM, it is possible to have a larger branching ratio for $H \rightarrow b \bar{b}$ and also a larger $W H$ production cross-section. For example, in MSSM there are two Higgs doublets $H_{u}$ and $H_{d}$ giving up- and down-quark masses, respectively. The Yukawa coupling of $b$ quark to the SM-like Higgs is scaled by a factor $\tan \beta=v_{u} / v_{d}$. Here $v_{i}$ is the vacuum expectation values of $H_{i}$. In the MSSM $\tan \beta$ can be as large as a few tens. The $\sigma \cdot \operatorname{BR}(H \rightarrow b \bar{b})$ can be enhanced. The same can be said for many multiHiggs models. The enhanced coupling also helps to make the total cross-section enhanced. These models also have charged Higgs contributions. If the mass of the charged Higgs is not too far away from the neutral ones, they can 
also enhance the total dijet excess. However, a universal enhancement in down quark sector may not be enough to have a large enough $W H$ production cross-section. To this end we note that there are models where the light quark Yukawa couplings can be enhanced even more, such as the private Higgs model and other Higgs multiplet models [3$5]$. In this type of models the total $W+$ dijet excess may become more close to the CDF value. This type of models usually also have large $Z H$ production which may be a problem. We will come back to this later.

The $W$ +dijet excess may also be due to new gauge bosons, such as a $Z^{\prime}$. There are stringent constraints on the mass of a new $Z^{\prime}$ gauge boson if it has significant coupling to charged leptons. Various data, for example, the Tevatron dilepton data, have constrained the mass to be much larger than the $150 \mathrm{GeV} / c^{2}$ resonant mass. However, if the $Z^{\prime}$ couples primarily to quarks [6-8], that is the $Z^{\prime}$ is hadrophilic or leptophobic, the constraints are much less severe. It is possible to have a low $Z^{\prime}$ mass to produce the $W+$ dijet excess. As we are studying this possibility, several papers [9-12] appeared showing that this is indeed possible if the coupling of the $Z^{\prime}$ to quarks is similar in order compared with the $U(1)_{Y}$ coupling. With such a large coupling, $s$-channel production of $Z^{\prime}$ can also become significant, but the signal may be buried under the large QCD background. With a hadrophilic $W^{\prime}$, it is also possible to explain the dijet excess.

In the above two classes of models, and also most of models relying on a new particle resonance $[9,13]$ to explain the CDF dijet excess data also predict excess for associated $(\gamma, Z)+$ dijet production. The reason is that the vertex at which a $W$ is emitted from a quark line, a replacement of $W$ by a $Z$ or a $\gamma$ is possible. If one starts with the same quark inside a proton, after the replacement, the quark which emits the new particle changes identity compared with the $W$ emission. This may change the crosssection significantly if the new particle couples to up and down types of quarks differently. However, there is also contribution from a different type of quark inside a proton after the replacement of $W$ by a $Z$ or a $\gamma$ the quark emitting the new particle is the same type of quark. Since both up and down types of quarks have sizeable non-vanishing parton distribution functions (PDF) inside a proton, the associated productions with a $W$ and a $Z$ or a $\gamma$ should have similar orders of magnitude. If there are other types of vertices, such as two gauge bosons to a new Higgs or a new gauge boson, the replacement of a $W$ by a $Z$ in the final state is also expected to have similar magnitude in strength. This is a generic prediction for a new particle interpretation of the CDF $W+$ dijet excess.

In fact, CDF has also investigated the shape of the dijet mass distribution in $Z+$ jets event. There is no statistically significant deviation from the SM expectation [1]. This disfavors the new particle interpretation. At present, one cannot rule out/in models with high confidence using this data. But this is something one needs to keep in mind when making claims. Future improved data can provide with more information. In the following we discuss an alternative possibility which is free from this potential problem. This has to do with dijet background from single top quark production.

There have been several interesting discoveries concerning some intriguing features of the nucleon which are beyond naive theoretical predictions. The difference between the EMC measured value of the spin-dependent structure function of the proton [14] and previous theoretical predictions triggered the proton spin "crisis" or spin "puzzle". It has been known that the relativistic effect due to transversal motions of quarks [15-19] and the sea content of the nucleon [20] play important roles to understand the proton spin problem. A number of models concerning the sea content of the nucleon, such as the baryon-meson fluctuation model [21] and the chiral quark model $[22,23]$, not only play significant roles to understand the proton spin structure [20,21,24], but also show their remarkable significance to explain the flavor asymmetry of the nucleon sea $[21,25-27]$ reported by the NMC Collaboration $[28,29]$. More interestingly, the baryon-meson fluctuation model and the chiral quark model have been also found to produce a strange-antistrange asymmetry of the nucleon, and such strangeness asymmetry with natural model estimations $[30-32]$ can explain the $\mathrm{NuTeV}$ anomaly $[33,34]$ of the deviation of the NuTeV measured value of weak mixing angle compared with other measurements. From the above discussion, we see that the intriguing features of the nucleon sea have played significant roles in the understanding of several anomalies beyond theoretical predictions.

Therefore, it is natural to examine the possible relevance of the nucleon sea with the CDF excess in $W+$ dijet production. The energy carried away by the produced $W$ boson should be larger than $100 \mathrm{GeV} / c^{2}$, and the dijet should carry more than $200 \mathrm{GeV} / c^{2}$ of energy. Thus the total energy of the produced $W+$ dijet event should be larger than $300 \mathrm{GeV} / c^{2}$, which means that more than $1 / 6$ of the total energy of the colliding proton and antiproton should be spent to produce such an event. This happens when at least one of the parton in the colliding proton and antiproton carried a momentum fraction larger than 0.1 , i.e., with Bjorken variable $x \geq 0.1$, a region where the intrinsic quarks (aniquarks) of the nucleon dominant.

It is important to distinguish between two distinct types of quark and gluon contributions to the nucleon sea measured in various deep inelastic processes: "extrinsic" and "intrinsic". The sea quarks generated from the QCD hard bremsstrahlung and gluon-splitting are referred as "extrinsic" quarks, since the sea quark structure is associated with the internal composition of gluons, rather than the nucleon itself. In contrast, sea quarks which are multi-connected to the valence quarks of the nucleon are referred to as intrinsic sea quarks. It has been shown [21] that the intrinsic quark-antiquark pairs generated by the minimal energy non-perturbative meson-baryon fluctuations in the nucleon sea provide a consistent framework to understand the nucleon intrinsic quarks. The model predicts an excess of intrinsic $d \bar{d}$ pairs over $u \bar{u}$ pairs, as supported by the Gottfried sum rule violation [26]. Furthermore, the meson-baryon fluctuations of the nucleon 
(a)

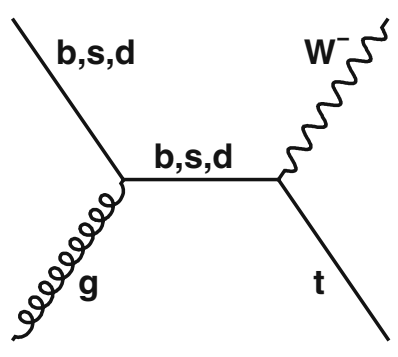

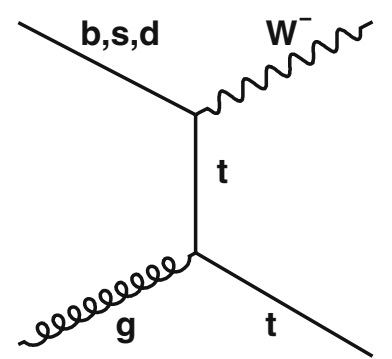

(b)
Fig. 1. The diagrams for the sub-processes of single top quark production associated with a $W$ boson production.

sea produce a striking quark/antiquark asymmetry in the momentum distributions for the nucleon strangeness, and such asymmetry provides a natural explanation [30] of the $\mathrm{NuTeV}$ anomaly within the standard model. The intrinsic quarks of the nucleon sea can be also alteratively modeled by the chiral quark model $[27,31]$, and all of the abovementioned anomalies can be also understood as well.

In Fock state wave functions containing heavy quarks, the minimal energy configuration occurs when the constituents have similar rapidities. Thus one of the most natural features of intrinsic heavy sea quarks is their contribution to the nucleon structure functions at large $x$ in contrast to the small $x$ heavy quark distributions predicted from photon-gluon fusion processes. This feature of intrinsic charm [35-38] has been extensively discussed. The extension of the intrinsic quark idea to the intrinsic bottom has been also studied [39]. The intrinsic bottom can contribute to the production of a $W$ boson plus a single top which can decay into two jets [40]. Therefore the intrinsic quarks of the nucleon do have relevance to the CDF excess of $W+$ dijet events.

The situation can be illustrated in fig. 1, where an intrinsic bottom (anti-bottom) quark in the colliding proton (or antiproton) transforms into a $W$ boson and a top (anti-top) quark when interacting with a gluon in another colliding particle. The produced top can contribute to the dijet detected by CDF. As some particles might be missing in the final re-constructed jets, the CDF excess of dijets in the range $120-160 \mathrm{GeV} / c^{2}$ does not exclude the contribution from decaying of a single top into two jets. The predicted rate for $W+t$ single top quark production at the Tevatron collision energy of $1.96 \mathrm{TeV} / c^{2}$ is $0.28 \pm 0.06 \mathrm{pb}$ for a top quark mass of $172.5 \mathrm{GeV} / c^{2}$ [40]. Such a contribution has been considered as a single top background in the CDF analysis of $W+$ dijet events.

The intrinsic light-flavor down-type quarks, i.e., $d$ and $s$ quarks of the nucleon intrinsic sea might also contribute to the $W+t$ single top quark production if one replaces the $b$ quark in fig. 1 by a $d$ or an $s$ quark. Such contributions might be highly suppressed due to the rather small values of quark mixing elements $V_{t d}=$
$0.00874_{-0.00037}^{+0.00026}$ and $V_{t s}=0.0407 \pm 0.0010$ compared with $V_{t b}=0.999133_{-0.000043}^{+0.000044}[41]$. However, as the probability of finding intrinsic bottom quarks is also highly suppressed compared with those of finding $d$ and $s$ quarks, we need to check their relative ratios between each other from rough estimates. Unlike "extrinsic" quarks, which are rather "soft" at small $x$ due to productions of the QCD hard bremsstrahlung and gluon-splitting, the "intrinsic" quarks of the nucleon can be rather "hard" at larger $x$. As it is rather difficult to distinguish between $d$ and $s$ quarks in the extraction of flavor-dependent quark distributions, and also the intrinsic $s$ quark distribution is predicted to be harder than the intrinsic $\bar{s}$ quark distribution in the baryon-meson fluctuation model, the available parametrizations of parton distribution functions may have underestimated the strange quark distribution at larger $x$. Therefore we need to estimate the relative contributions of $d, s$ and $t$ quarks from qualitative analysis.

The relative ratios of finding intrinsic $s$ and $b$ quarks inside a proton can be estimated from their off-shell-ness from the ground nucleon $[35,36]$, therefore

$$
\frac{\operatorname{Probablity}(s)}{\operatorname{Probablity}(b)} \sim \frac{m_{b}^{2}}{m_{s}^{2}}=\left(\frac{4.67 \mathrm{GeV}}{101 \mathrm{MeV}}\right)^{2} \approx 2 \times 10^{3} .
$$

The relative suppression of $s$ versus $b$ contributions to $W+t$ production due to the quark mixing can be also estimated

$$
\left(\frac{V_{t s}}{V_{t b}}\right)^{2}=\left(\frac{0.0407}{0.999133}\right)^{2} \approx 1.7 \times 10^{-3} .
$$

Therefore the ratios of $s$ versus $b$ quark contribution to $W+t$ production is 3.6, which corresponds a rate for $W+t$ single top quark production from $s$ quark contribution of being $1 \mathrm{pb}$. As $s(x) / \bar{s}(x)$ ranges between $1.5 \rightarrow 5$ for $x \geq 0.1$ (see fig. 5 of ref. [32]), we can therefore suspect that the $s$ quark contribution is dominant than $\bar{s}$ quark for the $W+t$ single top production for the colliding proton. This may also enhance the intrinsic strange quark contribution to the $W+t$ production by a factor of $2 \rightarrow 3$, corresponding to $2 \rightarrow 3 \mathrm{pb}$. Considering that the above estimate is very rough, should be reasonable in the order of magnitude, we thus can conclude that the intrinsic strange quark of the nucleon has a non-negligible contribution to the CDF excess of $W+$ dijet events.

We need also an estimate on the contribution from $d$ quarks. There is an additional valence $d$ quark inside the proton besides the intrinsic $s$ quarks at larger $x$. As the intrinsic $s$ quark is of $1 / 3$ of the sea $d$ quark distribution as in previous data analysis [32], we can thus use a larger probability of 5 times of finding a $d$ quark than an $s$ quark at larger $x$. The $d$ quark contribution to $W+t$ production is highly suppressed due to the quark mixing

$$
\left(\frac{V_{t d}}{V_{t b}}\right)^{2}=\left(\frac{0.00874}{0.999133}\right)^{2} \approx 7.7 \times 10^{-5} .
$$


The rate for $W+t$ single top quark production from $d$ quark contribution is thus estimated to be $0.16 \mathrm{pb}$, which is one half compared with the intrinsic bottom contribution. Thus the $d$ quark contribution to $W+t$ single top quark production should be also included as a background in the Tevatron $W+$ dijet analysis.

For the intrinsic strange quarks, the strangeantistrange asymmetry is a remarkable feature which is predicted from theory [21] and is further found to provide a viable explanation [30-32] of the $\mathrm{NuTeV}$ anomaly. Here we find that the strange quark has an important contribution to the $W+t$ single top production at the Tevatron collider of proton and antiproton collision. Thus the Tevatron $W+t$ production can also offer an ideal process to study the strange-antistrange asymmetry of the nucleon sea with unique advantages. As strange quarks are more "hard" than antistrange quarks inside the proton, we thus can predict that the $W^{-}+t$ events with $W^{-}$boson plus single top quark production should occur more likely in the proton forward direction, whereas the $W^{+}+\bar{t}$ events with $W^{+}$boson plus single antitop quark production should occur more likely in the antiproton forward direction. We thus can use the $\left(W^{-}+\mathrm{t}\right) /\left(W^{+}+\bar{t}\right)$ asymmetry to extract information on the strange-antistrange asymmetry of the nucleon. Of course, a more quantitative analysis is needed for confrontation with experimental observation.

One interesting feature of the CDF results is that there is no anomaly in the dijet mass distribution of $Z+$ jets events. A reasonable explanation for the CDF excess of $W+$ dijet events should be able to accommodate this feature also. Our suggestion to use the intrinsic strange contribution to $W+t$ single top quark production for the $W+$ dijet excess can accommodate this feature naturally. There is no quark-flavor-changing process in $Z$ production, therefore no single top production can be resulted from intrinsic strange and bottom quarks. Thus no excess of dijets with mass around $150 \mathrm{GeV} / c^{2}$ can be found in associated $Z$ +jets production in our explanation. This is a distinctive feature than those new particle resonance models. More precise data in future can distinguish different mechanisms for the CDF dijet excess.

In summary, we analysed the CDF observed excess in the production of two jets in association with a $W$. If this excess is interpreted as associated $W$ production with a new particle of mass $150 \mathrm{GeV} / c^{2}$, such as a Higgs or a $Z^{\prime}$ gauge boson, which decays into two jets, there should also exist dijets excess with an associated $Z$ production. CDF data, however, do not show statistically significant deviation from the SM expectation for $Z$ plus dijets events. This disfavors the new particle explanation. This therefore led us to examine the nucleon intrinsic quark contributions to the $W+t$ single top quark production. We found that the intrinsic strange quarks can provide an important contribution to the $W+t$ single top quark production, with no enhancement in the production of $Z+$ jets events. The $W+t$ production contributes to the CDF $W+$ dijet events, thus we provide a possible explanation for the CDF excess of $W+$ dijet events. We also provide a prediction of $\left(W^{-}+t\right) /\left(W^{+}+\bar{t}\right)$ asymmetry in the Tevatron proton and antiproton collision. Thus our suggestion of a larger intrinsic strange contribution to the $W+t$ single top quark production can be tested by detailed analysis of data.

This work is partially supported by NSC, NCTS, NSFC (Grants No. 11021092, No. 10975003, No. 11035003, and No. 11120101004), SJTU 985 grant. BQM acknowledges the support of NCTS North and warm hospitality from W.-Y. Pauchy Hwang and Pei-Ming Ho during his visit of NTU. We also acknowledge the stimulating discussions with Sang Pyo Kim and Miao Li.

Open Access This article is distributed under the terms of the Creative Commons Attribution Noncommercial License which permits any noncommercial use, distribution, and reproduction in any medium, provided the original author(s) and source are credited.

\section{References}

1. T. Aaltonen et al., Phys. Rev. Lett. 106, 171801 (2011).

2. T. Altonen et al., Phys. Rev. Lett. 103, 101802 (2009).

3. R.A. Porto, A. Zee, Phys. Lett. B 666, 491 (2008).

4. S.-L. Chen, N.G. Deshpande, X.-G. He, J. Jiang, L.H. Tsai, Eur. Phys. J. C 53, 607 (2008).

5. X.-G. He, L.-H. Tsai, Eur. Phys. J. C 71, 1598 (2011).

6. P. Chiappetta, J. Layssac, F.M. Renard, C. Verzegnassi, Phys. Rev. D 54, 789 (1996).

7. K.S. Babu, C.F. Kolda, J. March-Russell, Phys. Rev. D 54, 4635 (1996).

8. V.D. Barger, K.-m. Cheung, P. Langacker, Phys. Lett. B 381, 226 (1996).

9. K. Cheung, J. Song, Phys. Rev. Lett. 106, 211803 (2011).

10. M.R. Buckley, D. Hooper, J. Kopp, E. Neil, Phys. Rev. D 83, 115013 (2011).

11. F. Yu, Phys. Rev. D 83, 094028 (2011).

12. X.P. Wang, Y.K. Wang, B. Xiao, J. Xu, S.h. Zhu, Phys. Rev. D 83, 117701 (2011).

13. E.J. Eichten, K. Lane, A. Martin, Phys. Rev. Lett. 106, 251803 (2011).

14. J.J. Aubert et al., Phys. Lett. B 110, 73 (1982).

15. B.-Q. Ma, J. Phys. G: Nucl. Part. Phys. 17, L53 (1991).

16. B.-Q. Ma, Q.-R. Zhang, Z. Phys. C 58, 479 (1993).

17. B.-Q. Ma, Phys. Lett. B 375, 320 (1996).

18. B.-Q. Ma, A. Schafer, Phys. Lett. B 378, 307 (1996).

19. B.-Q. Ma, I. Schmidt, J. Soffer, Phys. Lett. B 441, 461 (1998).

20. B.-Q. Ma, I. Schmidt, J.-J. Yang, Eur. Phys. J. A 12, 353 (2001).

21. S.J. Brodsky, B.-Q. Ma, Phys. Lett. B 381, 317 (1996).

22. A. Manohar, H. Georgi, Nucl. Phys. B 234, 189 (1984).

23. S. Weinberg, Physica A 96, 327 (1979).

24. T.P. Cheng, L.-F. Li, Phys. Rev. Lett. 74, 2872 (1995).

25. E.J. Eichten, I. Hinchliffe, C. Quigg, Phys. Rev. D 45, 2269 (1992).

26. Y. Ding, B.-Q. Ma, Phys. Rev. D 73, 054018 (2006).

27. H. Song, X. Zhang, B.-Q. Ma, Phys. Rev. D 82, 113011 (2010).

28. P. Amaudruz et al., Phys. Rev. Lett. 66, 2712 (1991).

29. M. Arneodo et al., Phys. Rev. D 50, R1 (1994).

30. Y. Ding, B.-Q. Ma, Phys. Lett. B 590, 216 (2004). 
31. Y. Ding, R.-G. Xu, B.-Q. Ma, Phys. Lett. B 607, 101 (2005).

32. Y. Ding, R.-G. Xu, B.-Q. Ma, Phys. Rev. D 71, 094014 (2005).

33. G.P. Zeller et al., Phys. Rev. Lett. 88, 091802 (2002).

34. G.P. Zeller et al., Phys. Rev. D 65, 111103 (2002).

35. S.J. Brodsky, P. Hoyer, C. Peterson, N. Sakai, Phys. Lett. B 93, 451 (1980).

36. S.J. Brodsky, C. Peterson, N. Sakai, Phys. Rev. D 23, 2745 (1981).
37. B.W. Harris, J. Smith, R. Vogt, Nucl. Phys. B 461, 181 (1996).

38. J. Pumplin, H.L. Lai, W.K. Tung, Phys. Rev. D 75, 054029 (2007).

39. J. Pumplin, Phys. Rev. D 73, 114015 (2006).

40. A. Heinson, T.R. Junk, arXiv:1101.1275 [hep-ex].

41. Particle Data Group (C. Amsler et al.), Phys. Lett. B 667, 1 (2008). 